\title{
Acoustic Imaging in Materials Evaluation
}

\author{
W. Arnold \\ Fraunhofer Institute for Non-Destructive Testing (IzfP) \\ Saarbrücken, Germany
}

Acoustic imaging has profited greatly from advances in the theoretical understanding of contrast mechanisms, and from the improved availability of high-speed computers which allows one to model and interpret the complex contrast mechanisms and to handle the large amount of data involved.

Contrast in acoustic imaging techniques is mainly determined by the elastic properties of the sample with which the waves interact. Since these waves penetrate opaque materials, it is possible to image internal structures, to look for defects and to gain information about elastic properties - features which make these techniques important tools in non-destructive evaluation (NDE) and materials characterization. Acoustic imaging at low frequencies ( $<10 \mathrm{MHz}$ ) is mostly used to simply detect defects in a given volume - an application of great practical importance. Working at high frequencies reveals more detail about the microstructure of materials because scattering, absorption and dispersion due to defect agglomerates, second phases, dislocations, and inhomogeneities become more significant.

Theories underlying acoustic imaging cover, in principle, both frequency regimes. At present, however, these theories focus on defect sizing and characterization (for example, whether a crack or inclusion is present in a component). The effects of attenuation and dispersion in the theories become more significant as the frequency $v$ increases because their contributions increase with $v^{2}$, as does the density of scattering features which might be present. I shall therefore concentrate on imaging at frequencies above $10 \mathrm{MHz}$ in reviewing the applications of acoustic imaging methods for materials evaluation.

Walter Arnold heads the Research Group on Acoustics and Optics for materials characterization at the Fraunhofer Institute for Non-Destructive Testing, Universität, W-6600 Saarbrücken 11, and teaches in Saarbrücken's engineering faculty. He studied physics at the Technical University, Munich, and was a postdoc at the CNRS low temperature lab, Grenoble, and IBM, Yorktown Heights, before working as a research scientist at the MPI, Stuttgart, and with Brown Boveri.

\section{High Frequency Techniques}

Various types of equipment, both commercial and under development, are available for acoustic imaging:

1) The scanning acoustic microscope (SAM) which is analogous to the optical microscope [1] as it uses focussed acoustic waves [see Gremaud G. et al., page 167].

2) The scanning laser acoustic microscope (SLAM) where ultrasonic plane waves travel through the sample [2]: internal structures modify the sound field by scattering and absorption according to their elastic and geometric properties. Surface distortions caused by the sound field are transmitted to a light reflecting layer coupled acoustically to the sample surface by water and are detected with a knife-edge scheme (Fig. 1). As both amplitudes and phases of the surface distortions are measured its is possible to obtain separately the real and imaginary parts of the sound field. These form an acoustic hologram because the scattered and incident sound fields are both present in the image [3], rendering possible the reconstruction of defects by acoustic holography techniques [4].

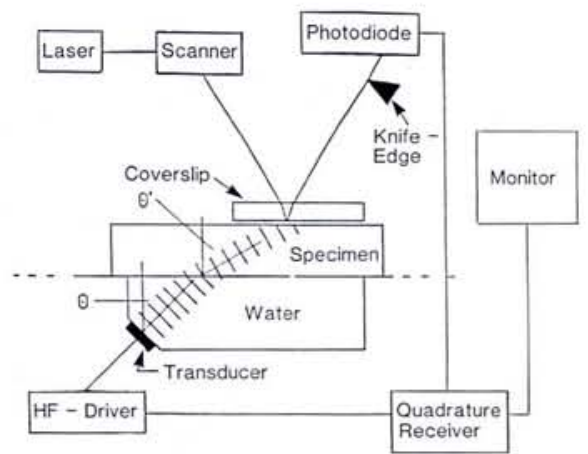

Fig. 1 - Principle of scanning laser acoustic microscopy (SLAM). The cover slip coupled acoustically to the sample under inspection is made of a plastic material with one side metallized to allow the acoustic imaging of non-reflecting materials. Sound waves travel through the sample and surface distortions on the reflecting surface are detected with a knife-edge scheme. The images of the surface obtained are acoustic holograms equivalent to Gabor holograms in their original form. Depending on the velocity of sound in the sample and the incident angle $\theta$, the wave generated by the transducer may be either refracted or mode-converted and emanate at an angle $\theta$. A quadrature is used to for detecting the phase of the image required for holographic reconstruction. Images are obtained in real time i.e. at 25 frames $/$.
3) High frequency acoustics is mostly employed to characterize advanced materials by detecting small defects, and evaluate their microstructures. For tions imply one must aim to detect defects smaller than $100 \mu \mathrm{m}$ in engineering ceramics by NDE techniques. Owing to the high velocity of sound in such materials, high frequencies are required. However, ultrasonic attenuation may become quite large at frequencies above $50 \mathrm{MHz}$, thus limiting the penetration depth. The other consideration is the spatial resolution: ultrasonic pulses shorter than $100 \mathrm{~ns}$ are needed in order to obtain sufficiently high values, and this calls for large bandwidths for both the electronics and the transducer. The transducer may be excited either with (broaponentially decaying step pulse as shown in Fig. 2, by an RF carrier pulse (narrowband excitation) [5].

Considerable new insight can be obtained by portraying an image in threedimensions instead of simply using two-dimensional representation to signal the presence of a defect at a given depth in the volume scanned. Applications in NDE of a system we have developed for doing this will be described below.

Scanning Acoustic Microscopy (SAM) The principle and theory of scanning acoustic microscopy is reviewed by Gremaud G. et al. on page 167 so we shall only describe an example of an NDE application where a quantitative evaluation of defect populations is sought. An increased resistance to crack growth with increasing crack done in electronic systems for NDE, or, 


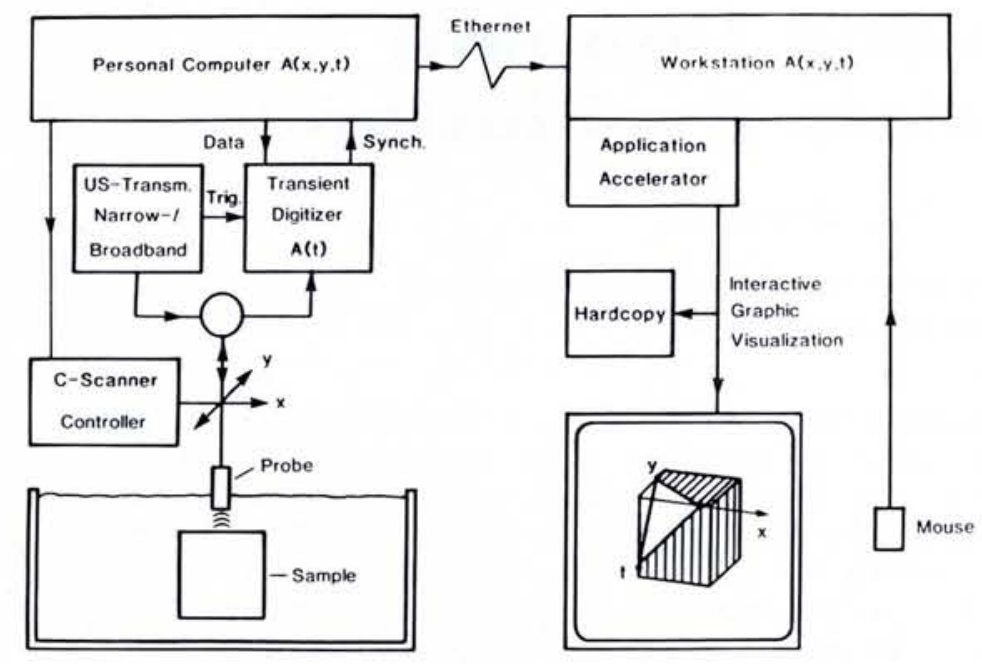

Fig. 2 - Block diagram of the author's high frequency acoustic set up. The apparatus comprises an electronic system for exciting the transducer (broadband system - Krautkrämer, Cologne-Hürth; narrowband - IzfP, Saabrücken), a scanning system and a digitization scheme allowing the three-dimensional viewing of data.

length is observed in some coarse-grained monolithic ceramics such as alumina (R-curve behaviour) [6]. Amongst the mechanisms which are postulated to contribute to this phenomenon in alumina is energy dissipation by the formation of microcracks in a $100 \mu \mathrm{m}$ damage zone around the macrocrack. As microcrack formation gives rise to a reduction in all elastic moduli, it should be possible to study the process by measuring the appropriate sound velocities in materials exhibiting R-curve behaviour.

The results of Rayleigh wave velocity $v_{\mathrm{R}}$ measurements at $1 \mathrm{GHz}$ for a notched alumina sample using the SAM $V(z)$ technique (where the amplitude of the reflected signal $V$ is measured as a function of the distance $z$ of the SAM lens from the specimen), with scanning across the crack working from the notch towards the crack tip, are shown in Fig. 3. Obviously, $v_{R}$ is reduced relative to the undamaged material close to the crack tip. However, with increasing distance from the notch, the reduction of $v_{R}$ becomes larger and two different damage zones appear. Zone I closest to the crack wall has a maximum reduction of $10 \%$ in the surface modulus at the tip (dotted lines in the figure): the size of this zone is $\approx 100 \mu \mathrm{m}$. Zone II (dashed lines) with a size of $\approx 400 \mu \mathrm{m}$ shows a constant $v_{R}$ which is $5 \%$ smaller than the velocity in the damaged material. So the predicted decrease in the sound velocity is indeed found, but further studies are necessary in order to elucidate the relative importance of all possible contributions to the R-curve behaviour. It should be emphasized that such measurements are difficult to carry out but, in general, only is in reality. The acoustic holography techniques used to reconstruct the de- fect are two-step processes. First, the amplitude and phase of the acoustic field emanating from the sonified object is detected in a plane adjacent to its surface. Second, from these field data, the scattered field in the defect plane can be reconstructed with a resolution of one wavelength. The relation between the fields $\phi(x, y ; 0)$ and $\phi(x, y ; \Delta z)$ in two parallel planes in the sample separated by a distance $\Delta x$ is given by a linear filtering process:

$\widetilde{\phi}\left(k_{x^{\prime}} k_{y} ; \Delta z\right)=\widetilde{\phi}_{0}\left(k_{x}, k_{y} ; 0\right) \exp \left(i k_{z} \Delta z\right)$ (1) with $k_{z}=\sqrt{ }\left(k^{2}-k_{x}{ }^{2}-k_{z}{ }^{2}\right)$ and $\tilde{\phi}$ the corresponding fields in $k$-space. $k_{x}$ and $k_{y}$ can be interpreted as the $x$ - and $y$-components of a plane wave with amplitude $\phi_{0}$. The field distributions between the detection plane and the object can therefore be obtained by calculating the two-dimensional Fourier transform of the field $\phi$, multiplying it by the filter function $\exp \left(-i k_{z} \Delta z\right)$ and Fourier backtransforming it into spatial coordinates (a process called back-propagation). Since outside $k_{x}{ }^{2}+k_{z}{ }^{2}=k^{2}$ the filter function is steeply increasing [4], the filtering is restricted to the inner face of the circle with radius $k$ to reduce noise. However, the signal-to-noise ratio (SNR) in the back-propagated image can be enhanced by deconvoluting the field data with the transform function of the laser detection system employed in SLAM, resulting in an improvement in the SNR of $\approx 10 \mathrm{~dB}$ compared to the original image.

\section{An experimental example}

Fig. 4 shows the SLAM image of an iron inclusion in a $\mathrm{SiC}$ bend-test bar ob-

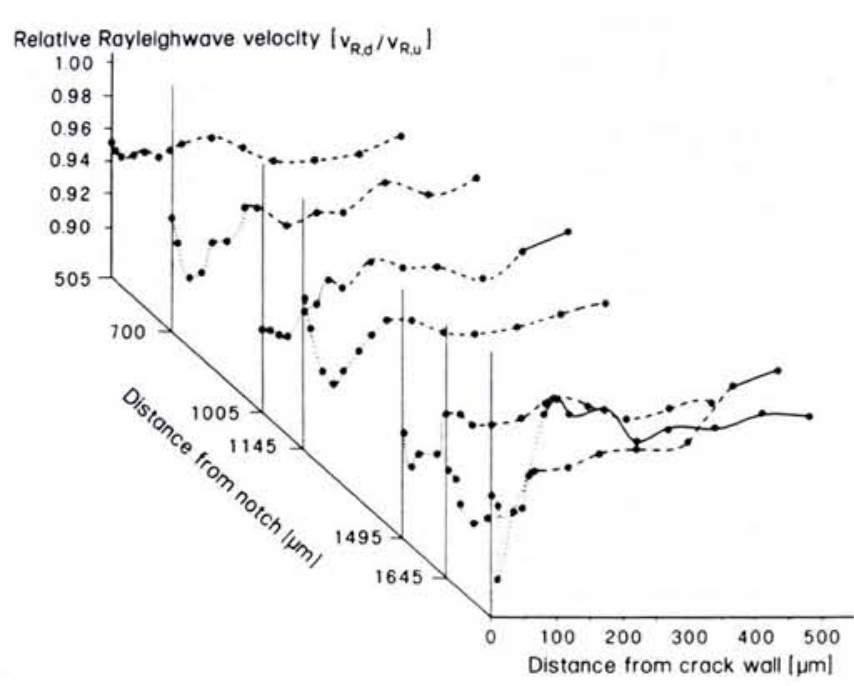

Fig. 3 - An application of SAM in non-destructive evaluation. Measured reduction of the Rayleigh velocity as obtained by evaluating $V(z)$ curves of a SAM (Leica, Wetzlar) along a macrocrack of $1.9 \mathrm{~mm}$ in length in a notched, double cantilever, fracture toughness test specimen of an alumina ceramic with a median grain size of $30 \mu \mathrm{m}$ : two damage zones are resolved (I - dotted lines; II - dashed lines). 
tained at $100 \mathrm{MHz}$. Diffraction of the sound field waves at the inclusion causes concentric ring patterns. On calculating the field distributions in planes of increasing depth, an image with an apparently optimal defect contrast is obtained for a section corresponding to the depth of the defect.

\section{High Frequency Acoustics}

Depending on the ratio of the defect size $a$ to the working wavelength, one can distinguish three regimes important for defect detection: Rayleigh scattering $(a / \lambda<<1)$, resonance scattering $(a / \lambda \approx 1)$ and geometric scattering $(a / \lambda$ $>>1$ ). As mentioned above, attenuation limits the propagation of ultrasonic waves in engineering materials at frequencies above $50 \mathrm{MHz}$. If we employ $50 \mathrm{MHz}$ waves for ceramics and similar materials with sound velocities greater than $6 \mathrm{~mm} / \mu \mathrm{s}$ we are still in the Rayleigh regime for our $100 \mu \mathrm{m}$ defect, i.e. outside the $\lambda \approx$ a regime of maximum contrast.

Neglecting diffraction, the intensity I backscattered from a given spherical defect can be estimated as

$$
I=I_{0}[f(\pi)]^{2} \pi a^{2} \exp (-\alpha r) d \Omega
$$

where $d \Omega=\Delta F / 4 \pi r^{2}$ with ultrasonic

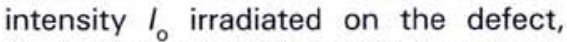
backscattered amplitude $f(\pi)$, attenuation coefficient $\alpha$, area of transducer $\Delta F$, depth of defect $r$ and solid angle $d \Omega$ subtended by the probe as seen from the defect. Because $f(\pi) \approx a^{2} v^{2}$ in the Rayleigh regime, $I / I_{0} \approx a^{6} v^{4}$ for an ultrasonic frequency $v$. This means that very efficient transducers with a high as possible centre frequency have to be employed in order to obtain a sufficiently large SNR for a given excitation voltage of the transducer.

Expressions similar to (2) also hold good for other defect shapes so it is necessary to design the electronic systems and probes such that the highest SNR is obtained and losses are minimized. At present, the detection limit of our set-up for defects is $30 \mu \mathrm{m}$ for inclusions at a few $\mathrm{mm}$ in depth, provided the ultrasonic attenuation in the test piece is less than $1 \mathrm{~dB}$ at $50 \mathrm{MHz}$. We have employed the system to evaluate materials for defects, lack of adhesion between different components, etc. [5].

\section{Three-dimensional slicing}

Considerable new insight can be obtained by representing the original data with a three-dimensional slicing technique. For the volume acquisition of data over the test volume we use a transient digitizer with eight-bit resolution and a sampling rate of $400 \mathrm{MHz}$. The full
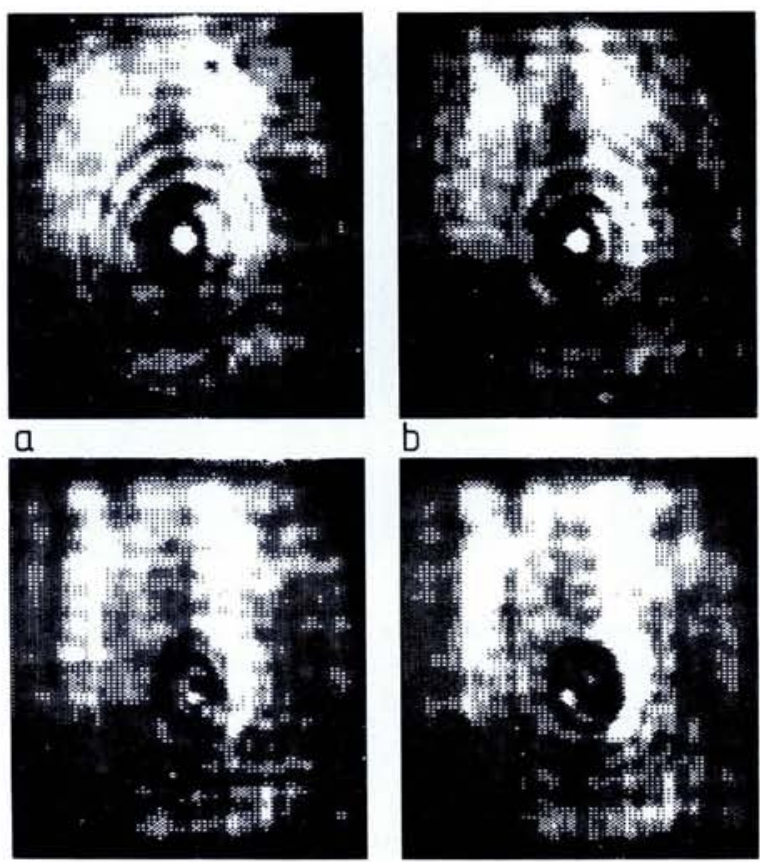

b

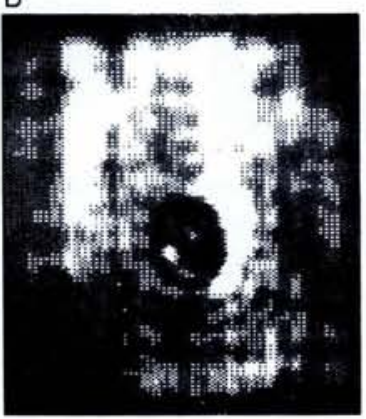

e

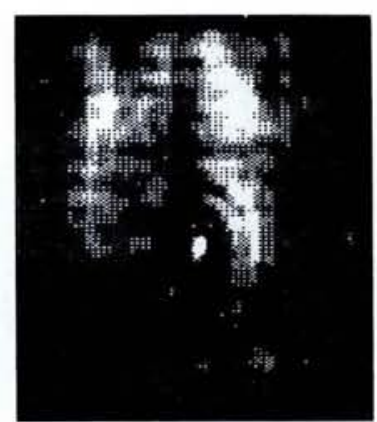

C

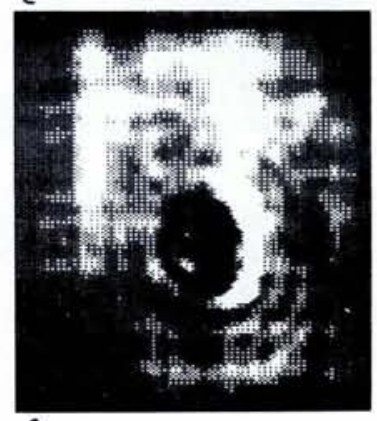

f

Fig. 4-SLAM image of a Fe inclusion in Si infiltrated SiC as obtained at the output detector (100 MHz working frequency; $2.8 \times 2.8 \mathrm{~mm}^{2}$ field of view). (a) Vertical distance from specimen surface $z=0$ (i.e. plane of detection is the specimen surface). (b-f) reconstructed images at increasing $z$ (steps of $200 \mu \mathrm{m})$ : the defect appears focussed in (c) and (d) indicating that it lay at a depth of approximately $500 \mu \mathrm{m}$.
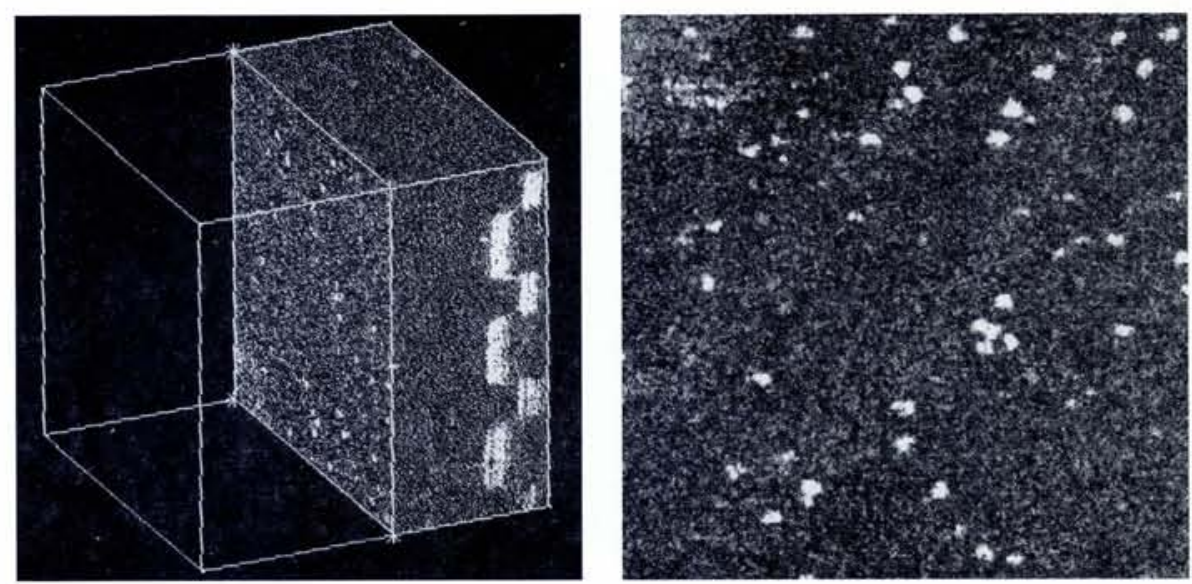

Fig. 5 - Volume representation of acoustic scattering data for a Si bonded SiC ceramic with three $0.5 \mathrm{~mm}$ deep vertical groves on its rear side. Slices perpendicular or parallel to the time axis, corresponding to conventional C-and B-scans, respectively, can be chosen.

amplitude detected by the ultrasonic transducer signal versus time of flight (the so-called A-scan) is digitized at each point of a meandering $x y$-scan of the component being inspected. The scanning system as well as the digitizer are controlled by software running on a personal computer (Fig. 2). A fast total measurement time has been achieved by optimizing data transfer from the digitizer to the PC. We digitize at 200 $\times 200$ points over the $x y$-plane and at 200 points in time, resulting in eight Mbytes of volume data which must be processed after acquisition to recons- truct defects or sample characteristics.

As an example, we present a volume measurement of a slab of a Si infiltrated $\mathrm{SiC}$ ceramic $\left(50 \times 50 \mathrm{~mm}^{2}\right.$, thickness $4 \mathrm{~mm}$ ), with three groves of $0.5 \mathrm{~mm}$ depth on its rear face, scanned using a $50 \mathrm{MHz}$ polymeric probe head (Krautkrämer, Cologne-Hürth, Germany) with broadband excitation. The scanned volume is represented in the left half of Fig. 5 by a cube in perspective, the lefthand surface of the cube being the $50 \times 50 \mathrm{~mm}^{2} x y$-scan plane, while the axis from left to right is the time (or depth) axis with length $2 \mu \mathrm{s}$. Ultrasound 


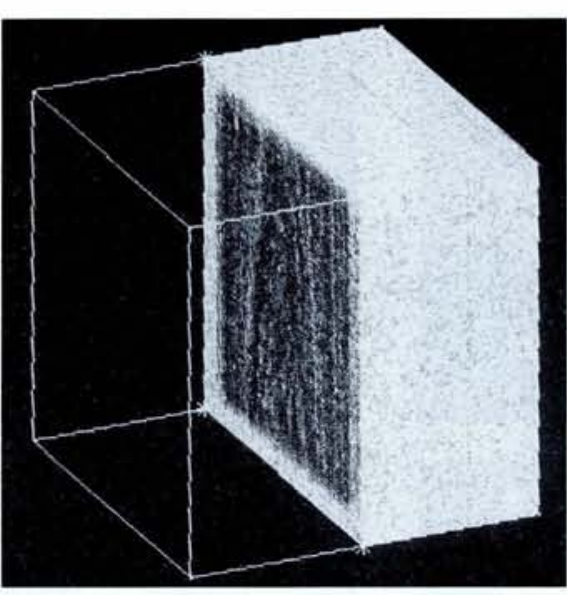

Fig. 6 - Frequency domain image of a powder metallurgical aluminium alloy component containing $2 \mathrm{vol}$. \% of $15 \mu \mathrm{m} \mathrm{Cu}$ particles. The flow pattern developed during the production process is visible owing to the particles' enhanced ultrasonic scattering power relative to the matrix.

was incident from the left side and absolute values of the amplitude are represented in the slicing technique by colour coded or grey scales (Fig. 5, right half).

Entrance and backwall echoes are visible at the left and right sides, respectively, of the front face of the cube. The cube can be turned around any axis by manipulating the mouse control of the $\mathrm{PC}$, and the interior of the sample can be inspected by moving one of the surface planes into the volume, thus making it possible to obtain equivalents to $B$ - and C-scans (see Fig. 5) in an easy and convenient way [8].

\section{Ray casting}

In the ray casting mode, image rays are sent through the volume and are projected onto the image plane (cover illustration). Depending on the imaging strategy, the maximum or the integrated ultrasound scattering amplitude along the ray is converted into a colour-coded value, leading to an X-ray like image. In a more sophisticated approach, different intervals of amplitude values are assigned to substances with selectable optical properties like colour or transparency. This artificial projection is illuminated by a movable light source to produce a realistic image. In a further step, many such views taken at different angles can be combined to give a video reconstruction.

\section{Transformed data}

The availability of the full time-dependence of the ultrasound signal allows one to apply data processing before visualization. For example, one can Fourier transform the received echo data and replace the time axis by a frequency axis. An image of such a volume then represents the spatial distribution

Acoustic imaging has clearly profited

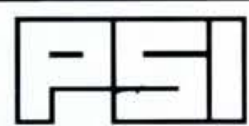

PAUL SCHERRER INSTITUT basis, the position of a

- participation in the SINQ project team

More detailed information can be obtained via

Paul Scherrer Institute,

CH-5232 Villigen PSI, Switzerland.

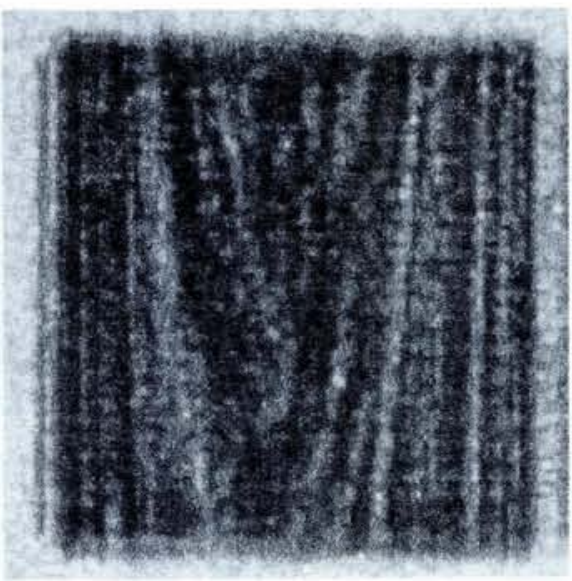

and in our understanding of the contrast mechanisms. There now exists a range of imaging methods suitable for use in non-destructive evaluation following our introduction of three-dimensional reconstruction capabilities in the conventional high frequency acoustic technique. In the future, we shall integrate our imaging software with programs for reconstructing defects using synthetic aperture techniques [9] and for controlling robotic scanning systems to allow the study of complex components.

\section{ACKNOWLEDGEMENTS}

I should like to thank R. Herzer, A. Morsch, U. Netzelmann, H.J. Pongratz, S. Pangraz, A. Quinten, and $\mathrm{H}$. Stolz for close collaboration, and $\mathrm{P}$. Höller and $M$. Kröning for encouragement and support.

\section{REFERENCES}

of the spectral power density of the selected echo at a certain frequency. We have studied a aluminium sample $\left(30 \times 30 \times 5 \mathrm{~mm}^{3}\right)$ manufactured by powder metallurgy in this way using data generated with a $50 \mathrm{MHz}$ transducer and broadband excitation. The slice (Fig. 6) representing the $25 \mathrm{MHz}$ component of the Fourier transformed backwall echo signal shows the typical flow structures of a two-phase material which arise during manufacturing. Contrast is more enhanced than in the conventional $\mathrm{C}$-image owing to frequency dependent scattering (Rayleigh scattering $\sim v^{4}$ ) by small copper particles.

\section{Future Developments} from advances in computer technology
[1] Quate C.F., Atalar A. and Wickramasinghe H.K., Proc. IEEE 67 (1979) 1092.

[2] Kessler L.W. and Yuhas D.E., Proc. IEEE 67 (1979) 526.

[3] Lin Z. et al., IEEE Trans. UFFC-34 (1987) 293.

[4] Morsch A. and Arnold W., in: Proc. 12th World Conf. on NDT; Eds J. Boogard and G.M. van Dijk (Elsevier Science Publishers) 1989.

[5] Pangraz S. et al., Proc. 18th Symp. Acoustical Imaging (1991); Eds. G. Wade and $\mathrm{H}$. Lee (Plenum Press), to be pub.

[6] See: Conference Reports of Forschungs zentrum (KFA) Jülich 7 (1990), Eds. H. Nickel and R.W. Steinbrech.

[7] Pangraz S. et al., Proc. 19th Symp. Acoustical Imaging (1992); Eds. H. Ermert and H.P. Harjes (Plenum Press), to be pub. [8] Netzelmann U. et al., in [7].

[9] Schmitz V., Müller W. and Schäfer G., in [7].

The Paul Scherrer Institute (PSI) in Würenlingen/Villigen, Switzerland, is a Swiss Federal Laboratory for multidisciplinary basic and applied research. One of its major experimental facilities, with pioneering character for the production of future intense neutron beams, the continuous spallation neutron source (SINQ), is now in a period of realization. For the neutron scattering group of PSI in collaboration with the laboratory for neutron scattering of the ETH Zurich, the Institute offers, on a contractual

\section{Scientist in Neutron Scattering}

preferably with experience in high resolution inelastic neutron scattering. The successful candidate should attend predominantly to the following functions:

- development of a neutron scattering instrument to be installed at SINQ

- scientific research in the field of solid state physics and materials science

SINQ-Secretariat, R. Bercher, Tel. +41 (56) 993402.

Applications which should include a curriculum vitae, the names and addresses of two referees and a list of publications should be sent to:

Würenlingen/Villigen, Personnel Division, Ref. code 3800, 